Revue internationale P.M.E.

Économie et gestion de la petite et moyenne entreprise

\title{
Un diagnostic de pré-démarrage pour les PME et PE
}

\section{André Archer}

Volume 3, numéro 3-4, 1990

URI : https://id.erudit.org/iderudit/1007987ar

DOI : https://doi.org/10.7202/1007987ar

Aller au sommaire du numéro

Éditeur(s)

Presses de l’Université du Québec

ISSN

0776-5436 (imprimé)

1918-9699 (numérique)

Découvrir la revue

Citer cette note

Archer, A. (1990). Un diagnostic de pré-démarrage pour les PME et PE. Revue internationale P.M.E., 3(3-4), 389-409. https://doi.org/10.7202/1007987ar

\section{Résumé de l'article}

Pour tenter de réduire les pertes en ressources de toutes sortes qu'accompagnent les insuccès des PME et PE, une approche dite de «pré-démarrage» est développée, et proposée à l'intention des entrepreneurs potentiels. Cette approche vise à éveiller l'entrepreneur potentiel aux différents écueils du marché et le porte à évaluer ses propres forces et faiblesses avant toute décision d'investir.

La méthode de "pré-démarrage«, testée auprès de deux échantillons tirés de deux secteurs de l'économie régionale de la Mauricie (Québec), montre comment elle peut contribuer à réduire les marges d'erreur des prévisions, éviter les maladresses etasseoir les décisions subséquentes sur des bases solides. 


\title{
Notes \\ Un diagnostic de pré-démarrage pour les PME et PE
}

\author{
André ARCHER* \\ GREPME \\ Université du Québec à Trois-Rivières
}

A Lise Richard

\begin{abstract}
RÉSUMÉ
Pour tenter de réduire les pertes en ressources de toutes sortes qu'accompagnent les insuccès des PME etPE, une approche dite de apré-démarrage" est développée, et proposéeà l'intention des entrepreneurs potentiels. Cette approche vise à éveiller l'entrepreneur potentiel aux différents écueils du marché et le porte à évaluer ses propres forces et faiblesses avant toute décision d'investir.

La méthode de upré-démarrage $\propto$, testée auprès de deux échantillons tirés de deux secteurs de l'économie régionale de la Mauricie (Québec), montre comment elle peut contribuer à réduire les marges d'erreur des prévisions, éviter les maladresses etasseoir les décisions subséquentes sur des bases solides.
\end{abstract}

* L'auteur avait un Ph.D. en économie agricole de l'Université de New York. Il était spécialiste en production sur les fermes laitières et en transformation agro-alimentaire. II a publié plusieurs articles et ouvrages sur le sujet. Il est décédé accidentellement le 22 novembre demier. Adresse : GREPME-UQTR, C.P. 500, Trois-Rivières (Québec), G9A 5H7. Premier manuscrit reçu : juin 1990. 


\begin{abstract}
In order to reduce losses in resources of all kinds due to the failure of PME and PE, an approach called " pré-démarrage" is developed and proposed to the potential entrepreneurs. The purpose of this approach is to prompt the potential entrepreneurs to consider carefully the forces at play in the market he intends to enter and evaluate his own forces and weaknesses before any decision to invest.

The method of «pré-démarrage", tested with two samples drawn from two sectors of the regional economy of the Mauricie (Québec), shows how it can contribute in reducing the margin of errors from previsions, in voiding mistakes and in strengthening future decisions.
\end{abstract}

\title{
RESUMEN
}

Para ensayar de reducir las pérdidas de toda clase que acompanan siempre el fracaso de las PME y PE, este estudio ha desarrollado a la intencion de los industriales potenciales, un planteamiento dicho de apre-lanzamiento". Su objetivo es de hacer resaltar los particularidades delmercado en que el emprendedor quiere desarollarse y ayudarle a determinar su vulnerabilidad o su solidez antes de invertir.

Este procedimiento, sometido a un test con dos muestras seleccionadas de dos sectores de la economia regional de la Mauricie (Quebec), ha mostrado como se puede contribuir a reducir los errores de las previsiones, a evitar torpezas y a mejorar las decisiones futuras. 


\section{Problématique}

Grâce à une structure d'organisation plutôt simple, qui leur confêre une très grande flexibilité d'ajustement et d'adaptation aux changements ainsi qu'une une aisance à innover et à créer des emplois, les petites et moyennes entreprises sont courtisées assidûment par les gouvemements confrontés aux problèmes de création d'emplois ou désireux d'accélérer le développement économique de leurs régions. Pour faciliter la tâche aux entrepreneurs potentiels, les pouvoirs publics ont conçu et mis en place - et cela à tous les niveaux — un ensemble d'organismes à vocation complémentaire, comme les conseils régionaux de concertation, les services municipaux de développement économique, les corporations de développement économique ou commissariats industriels travaillant de concert avec les associations des gens d'affaires, les chambres de commerce locales, etc., dont le rôle principal est la concertation pour favoriser la création d'entreprises. L'intervention de ces organismes s'est manifestée sous diverses formes : organisation d'incubateurs fournissant des locaux ou d'autres services, études de marché, préparation de dossiers, recherche de sources de financement, etc.

Pourtant, malgré tout cet encadrement et les aides reçues, on s'aperçoit que beaucoup de PME et de PE éprouvent d'énormes difficultés à se créer une dynamique pour assurer leur survie et leur développement. Fourcade (1986) constate que «si le nombre des petites et toutes petites entreprises s'est considérablement accru pendant la dernière décennie, la mortalité est particulièrement grande parmi elles durant les premières années d'existence». Selon Zimmermer et Scarborough (1988), le taux de survie des petites entreprises est extrêmement faible : $80 \%$ disparaissent au cours de leur première année d'existence et $92 \%$ après cinq ans. On pourrait alors se demander, pourquoi y a-t-il tant de PME qui se créent chaque année ? Il y a plusieurs raisons à cela. D'abord, les institutions publiques et para-publiques citées plus haut encouragent fortement le développement de l'entrepreneuriat dans le but bien évident de tirer avantage de la création d'emplois qui en découle. Ensuite, le désir de devenir son propre patron, lié à la conviction de pouvoir faire mieux que ceux qui ont échoué, et éventuellement faire aussi bien que ceux qui ont réussi, sont des motivations déterminantes pour l'entrepreneur potentiel. C'est aussi parce que le mécanisme du marché, son fonctionnement et la manière dont on peut en tirer parti sont analysés et évalués différemment selon les intervenants.

De toute façon, si ces échecs peuvent être interprétés comme de l'expérience accumulée qui pourrait, à la rigueur, être utilisée pour rectifier le tir des PME du même groupe en difficulté, ils n'en représentent pas moins un coût social à plusieurs égards. Les efforts consentis par les organismes gouvernementaux, les subventions accordées, même si leur vrai coût est difficile à estimer, sont une perte sèche du moins dans l'immédiat. De plus, du point de vue humain, la perte des épargnes accumulées, la blessure psychologique infligée par la fermeture d'une PME ou PE, les pertes d'emplois, l'effondrement du rêve de toute une vie sont difficiles à supporter même par les plus aguerris. 
Pourquoi en est-il ainsi ? Pourquoi le taux d'échec des PME est-il si élevé ? Plusieurs recherches sur les PME et PE partent de l'hypothèse que la motivation, l'esprit et le désir de réussite qui animent l'entrepreneur potentiel font de lui un entrepreneur solide dont le succès est presque garanti. Or, il n'en est rien. La plupart des problèmes concernant la structure du marché, l'intensité de la concurrence, les exigences de la clientèle, les caractéristiques des fournisseurs, leurs forces, leurs faiblesses, les liaisons qu'ils entretiennent avec d'autres marchés concurrents, les contraintes gouvernementales ne sont découverts qu'une fois que l'entrepreneur potentiel se trouve dans le feu de l'action, c'est-à-dire après qu'il eut amorcé le démarrage et consenti les investissements d'établissement. Une simple planification avant le démarrage est insuffisante pour permettre à l'entrepreneur potentiel de recueillir des informations factuelles, de les analyser et d'être bien sensibilisé à la structure de l'environnement économique dans lequel il désire évoluer. Il en faut plus.

La démarche proposée, caractérisée comme étant un diagnostic de prédémarrage, si elle est bien menée et si elle s'articule autour de faits et non de suppositions, éveilleral'entrepreneur potentiel en le rendant conscient des éventuels écueils auxquels il auraà faire face, le forceraà évaluer ses propres forces et faiblesses ainsi que celles de ses concurrents, le sensibilisera aux risques entourant sa décision et lui permettra de repenser ses stratégies et de décider, selon le cas, de persévérer, de reporter dans le temps certaines de ses actions ou peut-être de réévaluer ses attentes et de modifier ses objectifs. Bien sûr, cette démarche ne s'adresse pas à tous les types d'entrepreneurs, surtout pas à ces porteurs d'idées qui pensent que leur projet est sans faille et se croient donc bien armés pour affronter le marché avec tous les risques qu'une telle action comporte. Elle s'adresse plutôt aux pondérés, ceux qui considèrent le long terme, la croissance, le marché international, ceux qui recherchent les faits pour les analyser de manière réfléchie, restent ouverts aux suggestions, s'inspirent des expériences passées dans la mesure où ces dernières sont de nature à améliorer ce qui va être créé demain. Cette démarche peut aussi permettre aux organismes d'aides de mieux orienter ces expériences.

\section{Objectif}

La majorité des investissements encourus par les PME surtout lors du démarrage sont, en cas d'échec, irrécupérables ou presque car leurprix de récupération est, si tant est qu'il y ait échec, à toute fin pratique nul. A la lumière de cette constatation et pour tenter de réduire les pertes en ressources de toutes sortes qu'accompagnent les insuccès, cette étude se propose de développer, àl'intention des entrepreneurs potentiels définis précédemment comme pondérés, une démarche dite de "pré-démarrage». Que peut-on entendre par "pré-démarrage». Pour bien saisir le sens de cette expression, situons-nous par rapport à celui généralement accepté de «démarrage». Fourcade (1986) considère la phase de démarrage comme celle qui suit 
l'étape de la création et de l'installation : «débute au moment où l'entreprise lance la première série de fabrication commercialisée, ou bien reçoit les premières commandes». Le «pré-démarrage» serait alors la période qui précède le stade de création et d'installation. Plus spécifiquement, c'est une période exploratoire précédant la mise en train des opérations où les idées sont encore au stade de projet et où l'entrepreneur potentiel s'attèle à prendre des actions préventives visant à bien connaître son marché, le produit, les concurrents, avant toute décision importante engageant sa responsabilité. L'objectif de cette démarche est de scruter, de mettre à nu les particularités des marchés, de déterminer les liaisons inter-firmes concernant les échanges en amont (fournisseurs) et en aval (clients), d'évaluer la nature de la compétition entre les producteurs, les distributeurs; d'apprécier le rythme d'adoption des innovations dans la mise en marché du produit et de celui de la technologie dans la fabrication; de mesurer la nature et le rythme d'arrivée des substituts; d'expertiser les relations avec les pouvoirs publics, en un mot de passer au peigne fin l'environnement médiat et immédiat du secteur choisi par l'entrepreneur potentiel. Autrement dit, l'entrepreneur potentiel est soumis à un traitement similaire à celui de l'apprenti pilote d'avion de ligne. Celui-ci, avant d'être autorisé à prendre les commandes d'un avion, est placé en situation concrète jusqu'à ce qu'il puisse surmonter certaines difficultés et passer avec succès les différents tests jugés essentiels pour réussir dans ce métier. L'analyse de son environnement rend possible la collection de données et d'informations qui, soigneusement analysées, permettent à l'entrepreneur potentiel de déterminer sa vulnérabilité ou ses forces en même temps que celles de ses concurrents, d'identifier les créneaux inexplorés et d'envisager des stratégies de pénétration, en un mot de procéder à une exploration minutieuse de la plupart des liaisons que l'entrepreneur aura à entretenir avec son cadre d'évolution.

Cette approche précède toute autre démarche comme les études commerciales, techniques, financières préalables aux estimations prévisionnelles des capitaux d'investissement et des nécessités financières couvrant les fonds de roulement, de même que les frais d'établissement et de constitution. Elle a l'avantage, quand elle est bien menée, de contribuer à réduire les marges d'erreur de ces prévisions, d'éviter les maladresses et les carences organiques et d'asseoir les décisions subséquentes sur des bases solides.

\section{La démarche}

La démarche envisagée s'inspire de la notion de filières (J. Malsot, 1980; R. Tollet, 1982; B. Yon, 1983; A. Jacquemin et M. Rainelli, 1984; P. Garrouste, 1988; R. Arena et al., 1988; A. Torre, 1988) pour établir les liaisons de l'entreprise avec son environnement. Selon Torre (1988) en particulier, une filière «décrit les liaisons amont et aval entretenues par les branches de production». Elle établit les relations interindustrielles ou interfirmes à la manière d'un tableau input-output. Elle permet d'établir et de préciser le degré de force ou de faiblesse des rapports qu'une firme 
potentielle aura à entretenir avec son environnement. Pour Rainelli (1988), la filière peut être vue comme point de référence pour analyser les stratégies d'entreprises, en particulier pour évaluer les rapports d'échanges en amont et en aval et leurs conséquences sur le développement et l'avenir de la firme potentielle. Par ce biais, le contexte économique de l'entreprise est étudié sous l'angle micro-économique en mettant en évidence les activités de production de la phase initiale à la phase finale, les stratégies à développer à chaque étape et les relations à établir entre les intervenants en amont et en aval. Ensuite, l'environnement de l'entreprise est analysé sous l'angle méso-économique où le cadre s'élargit pour considérer, en plus des liaisons directes de l'entreprise, les relations que ses fournisseurs ou ses clients entretiennent avec d'autres agents du marché. Par exemple, l'entreprise potentielle serait intéressée à obtenir des informations sur le degré de vulnérabilité de son fournisseur vis-à-vis d'autres clients ou même du fournisseur de celle-ci. Le comportement des intervenants formant les liaisons immédiates dépend, dans une large mesure, des forces ou des faiblesses qu'ils ont vis-à-vis de leur propre environnement. Du point de vue stratégique, si un fournisseur est très vulnérable visà-vis de son environnement, il est peu fiable. L'entrepreneur averti doit donc, dans son plan d'action, envisager des fournisseurs substituts au cas où celui avec qui il fait affaire viendrait à faillir à son engagement.

Quels sont les éléments de l'environnement qu'on doit inclure dans le diagnostic?

Ceux-ci seront sélectionnés et analysés selon les critères information produit-marché-technologie. Ces critères tiennent bien compte des environnements de l'entreprise qui se conçoit et se créera éventuellement. Cette séquence définit les objectifs de l'entreprise et trace le chemin à parcourir pour y arriver. La collecte et l'analyse de l'information constituent l'essence de la stratégie de l'entrepreneur potentiel à ce stade du pré-démarrage. L'information qu'il doit recueillir touche le produit ou service, ses caractéristiques et ses spécifications, elle touche aussi les clients et leurs traits significatifs, les différents segments du marché à desservir; elle englobe enfin le choix de la technologie qui sera utilisée dans la production et la mise en marché du produit ou du service. L'information sera cueillie selon la triple perspective produit-marché-technologie et devra être traitée de manière à faciliter la prise de décision finale.

En considérant la firme comme un système ouvert, on peut voir qu'en amont sa mission est d'acquérir des ressources (matières premières, travailleurs, etc.), de procéder à leur transformation selon certaines normes légales, et, qu'en aval, d'offrir aux clients les services ou biens finis ou semi-finis ainsi obtenus. En plus des clients et des fournisseurs, son environnement comprend, en effet, un ensemble d'acteurs comme les firmes compétitrices, les agences gouvernementales, les banques, les consultants, les distributeurs, les agences de publicité, etc.

Avant de traiter un à un ces éléments, jetons un regard d'ensemble sur le secteur que l'entrepreneur potentiel a choisi. On tentera d'évaluer un point crucial qui 
conditionnera dès le départ la naissance et plus tard la survie de la firme. Il s'agit des barrières à l'entrée. Certaines sont visibles, comme le capital requis pour démarrer, les politiques gouvernementales et les réglementations en vigueur. D'autres le sont moins, tels les marques dominantes, le montant de publicité nécessaire pour se tailler une part de marché, l'accessibilité aux canaux de distribution et points de vente, etc.

L'identification des barrières à l'entrée comme les économies d'échelle à tous les niveaux (production, mise en marché, achats d'intrants, etc.), les capitaux nécessaires pour démarrer, l'accès aux canaux de distribution, les politiques gouvernementales, etc., est importante et particulièrement l'identification de celles qui - à prime abord - sont moins évidentes pour l'observateur mais qui apparaissent clairement à ceux qui oeuvrent sur le terrain. Ces barrières sont la plupart du temps particulières à un marché. Elles se sont élevées avec le temps à la faveur, soit des us et coutumes comme de différencier à tout instant le produit, soit de l'ancienneté de certaines firmes (image), soit du conservatisme des clients qui vouent leur fidélité à une marque donnée, soit des normes établies dans l'industrie qui consistent à offrir par exemple des marges de crédit aux clients, etc. Si elles sont présentes, il faut des ressources pour les surmonter. Aussi l'entrepreneur doit-il soigneusement déterminer leur importance et inclure dans ses prévisions les moyens et les stratégies pour pouvoir, le cas échéant, les franchir.

Considérons les fournisseurs. L'entrepreneur déterminera en tout premier lieu leur degré de concentration. Dépend-t-il de plusieurs fournisseurs indépendants ? Sera-t-il obligé d'acheter régulièrement un certain volume donné pour bénéficier de meilleurs prix ou dépend-t-il d'un consortium qui agit de concert ? La vulnérabilité de l'entrepreneur face à ses fournisseurs sera d'autant plus élevée que leur nombre sera réduit et leur concentration très forte.

Le nombre de fournisseurs d'un facteur donné peut être secondaire si la fonction de production tolère des intrants substituts. Ces intrants peuvent provenir de secteurs ou de marchés différents de celui dans lequel l'entrepreneur évolue. C'est donc un champ à explorer soigneusement et ce, pour parer à des situations d'urgence dans lesquelles le fournisseur régulier se révèle incapable de satisfaire à la demande.

Qu'en est-il des clients? Sont-ils concentrés ou indépendants? Quel est leur niveau d'éducation, leur âge, leur revenu ? La vulnérabilité de l'entrepreneur est proportionnelle au degré de concentration de ses clients. D'un autre côté, les produits substituts occupent-ils une part importante du marché ? Quel est leur degré de différenciation ? Les acheteurs visés sont-ils fidèles à certains substituts ou ont-ils une propension à magasiner ou à adopter des produits de remplacement ? La qualité du produit exerce-t-elle une influence particulière sur la clientèle visée ? Et cette qualité, de quoi dépend-t-elle ? De certains types de machines, d'ouvriers spécialisés? Est-ce que ces derniers sont faciles à trouver ?

La technologie est aussi un élément à considérer. Est-elle changeante ou traditionnelle ? Comment semble-t-elle affecter les coûts de production? La 
croissance et la survie des firmes du secteur dépendent-elles de l'adoption et de l'application des derniers cris de la technologie ? L'application de cette technologie augmente-t-elle la production au point d'entrainer des coûts fixes d'entreposage ? La rivalité entre firmes est-elle entretenue par la technologie qui contribue à faire baisser les coûts?

Un autre point qui mérite considération est la mise en marché du produit. On doit s'assurer de l'accessibilité des réseaux de distribution et des points de vente. De même, on appréciera l'importance qu'accordent les consommateurs aux marques, aux noms, et on évaluera le tout en terme de coûts.

Un dernier point non négligeable concerne les lois et réglementations des gouvernements. La production envisagée a-t-elle des effets sur l'environnement ? Si oui, à quel coût peut-on se conformer aux règlements en vigueur?

La réponseà chacune de ces interrogations sera pondérée aussi impartialement que possible par le décideur et, ce faisant, il pourra avoir une vision nette de la nature et des caractéristiques des différentes composantes de son secteur et savoir à quoi s'en tenir.

Une fois ce tour d'horizon complété, les faiblesses et les forces identifiées, il convient maintenant d'estimer le coût de toutes les démarches à entreprendre pour assurer le démarrage, si celui-ci se révèle possible. C'est alors qu'entrent en scène les sources de financement publiques et privées. Devra-t-on compter sur des avoirs personnels ou aura-t-on recours aux emprunts bancaires; pense-t-on s'adresser aux gouvernements pour des subventions ou des garanties de prêts ? Cet aspect de la question doit être abordé tant sous l'angle des multiples démarches à entreprendre que sous celui du temps requis pour obtenir des résultats.

Le diagramme qui suit montre le potentiel entrepreneur en A faisant face d'un côté aux différentes barrières à surmonter pour produire, et de l'autre à celles non moins importantes à franchir pour acheminer le produit de l'usine au client. Les points B,C,F,G,T représentent les partenaires avec lesquels il faut entrer en contact et les cercles symbolisent les différentes barrières à franchir.

Mais, comment peut-on obtenir les informations requises pour entreprendre la démarche proposée?

On dit souvent que le propriétaire d'une PME ou le potentiel entrepreneur n'a ni le temps, ni les ressources, ni la formation pour s'adonner à des études élaborées de marché ou de pré-démarrage. Soit. Mais a-t-il le choix ? L'enjeu est de s'informer quand il en est encore temps et de déterminer dans quelle direction on s'engage, ou alors d'investir à l'aveuglette avec tous les aléas que cela comporte.

La recherche de l'information pertinente est cruciale car sa valeur est directement proportionnelle au risque de prendre une mauvaise décision. Plus on peut disposer d'informations valables, plus la chance d'arriver à une bonne décision est élevée. 


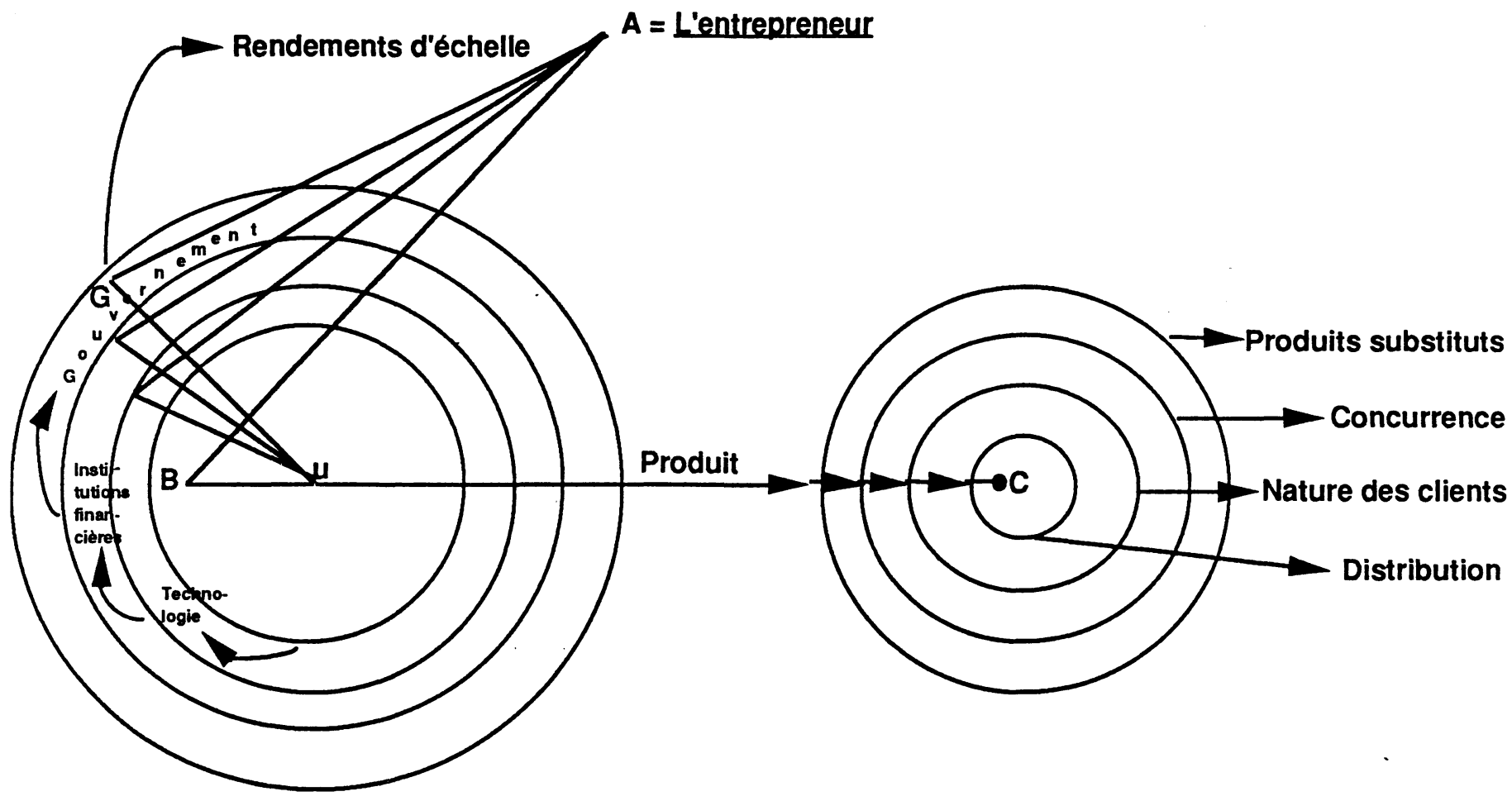

$. G=$ Gouvernement $. U=$ Usine $. C=$ Clients

$. F=$ Fournisseurs

$. T=$ Technologie

$. B=$ Institutions financières 
L'entrepreneur potentiel peut monter un dossier sur les composantes de son secteur en obtenant des informations de la part des clients du produit, des vendeurs, des banques, des chambres de commerce, des agences gouvernementales, etc. Notons en particulier que les vendeurs peuvent fournir des informations précieuses sur les particularités et la tendance générale des marchés.

Mais comment l'entrepreneur potentiel va-t-il organiser les informations recueillies pour arriver à une décision ?

Un bon nombre de méthodes de prise de décision sont à la mode. L'une d'elles, qui semble bien appropriée pour la question d'intérêt, est l'analyse QUID (Quantified Intrapersonal Decision Making :1987). Selon cette méthode, les questions au sujet desquelles on doit prendre une décision sont formulées clairement et les arguments pour ou contre sont relevés. Ces arguments sont pondérés selon les forces respectives des preuves accumulées en leur faveur. On utilise à cet effet une échelle ordinale de 1 à 8 où :

$$
\begin{aligned}
& 1=\text { mérite peu de considération } \\
& 2=\text { mérite considération } \\
& 3=\text { faiblement significatif } \\
& 4=\text { significatif } \\
& 5=\text { très significatif } \\
& 6=\text { important } \\
& 7=\text { très important } \\
& 8=\text { extrêmement important }
\end{aligned}
$$

On fait le total des scores enregistrés par les pour et contre à chaque question et on prend ensuite la moyenne correspondante. Sur la base de la différence entre les deux moyennes, on détermine si l'écart est assez significatif pour prendre une décision dans un sens ou dans un autre. Selon Moody (1983), il y a ambiguitté si la différence entre les moyennes est de 1 ou moins. Dans ce cas-là, toute décision doit être reportée jusqu'à ce que l'on dispose d'informations additionnelles. Ladifférence entre les deux moyennes doit être nettement supérieure à un seuil de 2 ou plus pour pouvoir permettre de prendre une décision tranchée.

Le tableau suivant peut être utilisé pour compiler les résultats des analyses et noter la pondération accordée à chaque élément. 


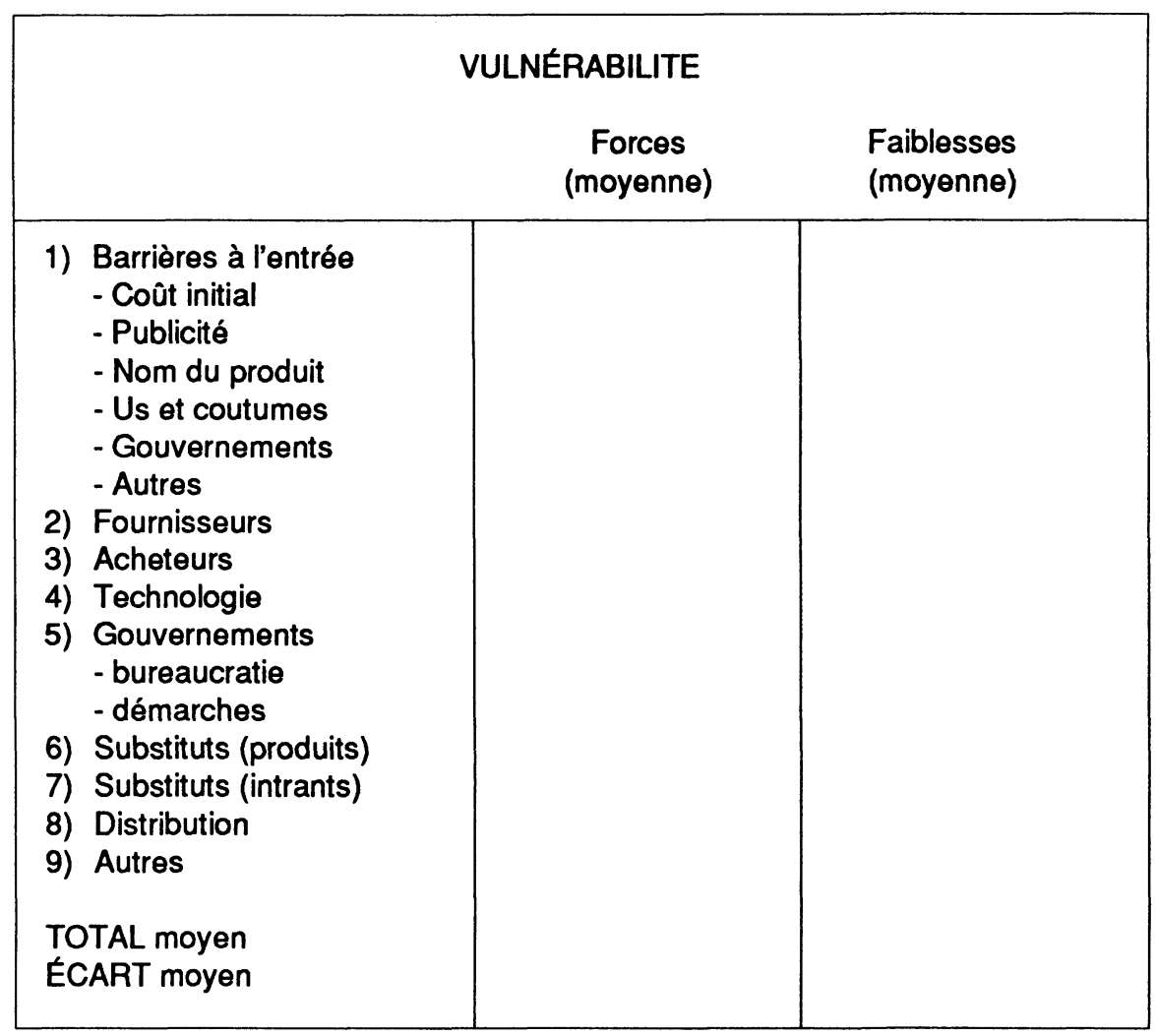

\section{Vérification du modèle}

Pour tester les idées exposées dans ce texte, un scénario a été appliqué en collaboration avec deux étudiants du programme de baccalauréat en économique pour explorer deux secteurs de l'économie régionale mauricienne: la fabrication des portes et fenêtres et l'imprimerie. Le choix de l'un de ces deux secteurs, l'imprimerie, a été fait sur la base que c'est avant tout un secteur de petites et très petites entreprises. Selon le Québec : mise en chiffres (1988), en 1987, on comptait 92 imprimeries dans la région de la Mauricie dont 41 avaient entre 1-4 employés, 13 entre 5 et 9 employés et aucune n'excédait 200-499 employés. Quant au secteur de la fabrication des portes et fenêtres, il a été exploré sur une base de complémentarité avec celui de l'imprimerie. En effet, une première prise de contact avec les deux secteurs avant le début de l'enquête proprement dite permet de relever certains traits bien typiques de chacun d'eux. Tandis que dans l'imprimerie on tendà retrouver des bricoleurs, des techniciens imaginatifs, ouverts aux innovations; dans les portes et fenêtres, on rencontre des gens plutôt conservateurs qui donnent l'impression de vouloir préserver la routine et la structure traditionnelle de leur métier. Dans l'imprimerie, les enfants achètent en 
général de leur père avec un capital modeste; dans les portes et fenêtres les entreprises sont plus capitalisées et toute la famille y travaille. Donc, ce sont des secteurs différents tant par leur structure de fonctionement que par la vision de leurs entrepreneurs. A la lumière desétapes énumérées dans la démarche de pré-démarrage et à l'aide d'un questionnaire spécialement conçuà cette fin, ces étudiants, lors d'une entrevue, recueillent des informations touchant aux barrières à l'entrée, aux fournisseurs, aux acheteurs, à la technologie, aux gouvernements, aux substituts du produit et aux intrants, à la distribution et à tous les autres points d'intérêt qui y sont soulevés et discutés. Un échantillon de dix(10) entreprises dans le secteur des portes et fenêtres et onze (11) entreprises dans le secteur de l'imprimerie est choisi aléatoirement. On constate que dans le secteur de l'imprimerie les entreprises sont assez récentes : trois ont entre 3-5 ans, quatre entre 6 et 10 ans, trois autres entre 11 et 16 ans et une seule a plus de 20 ans. Dans les entreprises de portes et fenêtres, seulement deux ont entre 8 et 10 ans, trois entre 15 et 20 ans et les autres, plus 20 ans. Pour compléter les données recueillies auprès des entrepreneurs en place, des renseignements additionnels sont obtenus auprès de quelques acheteurs et fournisseurs de ces entreprises ainsi que les points de vue fournis par deux institutions financières (une caisse populaire et une banque) sur l'opportunité, pour les nouveaux entrepreneurs désirant oeuvrer dans ces deux secteurs, d'obtenir du financement pour le fonctionnement de leurs entreprises.

L'analyse des résultats de ces démarches et les conclusions qui en découlent sont présentées ci-après.

Commençons d'abord par le secteur de la fabrication des portes et fenêtres : $30 \%$ des entreprises consultées ont de 15 à 20 employés, $40 \%$ de 21 à 35 employés et le reste entre 36 à 50 employés. En termes de chiffres d'affaires, $30 \%$ disent faire des affaires annuelles pour un montant allant de 0,5 à 1 million, $40 \%$ de 1 à 2 millions et le reste de 2 à 4 millions.

Considérons un à un les points du questionnaire. Le premier concerne les barrières à l'entrée.

La majorité des répondants $(80 \%)$ indique que l'entrée est plutôt difficile non à cause du capital fixe à investir, qui est de l'ordre de 500000 en moyenne, mais surtout à cause de l'importance du nom et de la réputation de certaines compagnies établies dans le secteur il y a 25 à 30 ans. De plus, le nouvel entrepreneur de ce secteur doit connaître la profession à fond, le marché, et s'assurer dès le départ d'une bonne clientèle pour garantir des entrées de fonds, car les déboursés du début étant surtout des coûts variables sont, de ce fait, plus élevés. De même, comme la ligne des portes et fenêtres connaît des mouvements cycliques substantiels, pour survivre, l'entrepreneur doit penser jumeler cette activité à d'autres comme la quincaillerie générale ou la pose des portes et fenêtres vendues aux particuliers. Enfin, tous les répondants disent que, compte tenu du grand nombre de fabricants, le marché est saturé de sorte que les chances de survie du nouvel entrant sont minces, à moins qu'il puisse innover soit sur la qualité du produit ou sur son prix de vente. 
Pour toutes ces raisons, la cote moyenne de -5 «très significatif» comme faiblesses est obtenue pour ce point.

Le deuxième élément considéré touche les fournisseurs des matières premières. Sur ce point, il ne semble pas y avoir de problème, si cen'est qu'il faut acheter un volume minimum pour bénéficier de prix avantageux. Par ailleurs, à cause des coûts de transport, on ne tire aucun bénéfice à acheter à d'autres provinces ou d'autres pays, même si les prix peuvent être meilleurs que ceux des fournisseurs locaux. La pondération moyenne de +4 «significatif» comme forces, est calculée pour ce point.

Le troisième point concerne les acheteurs. Plus de $75 \%$ des répondants considèrent qu'un minimum de publicité est nécessaire surtout en périodes de ralentissement des activités. Toutefois, tous sont d'accord sur le fait que la fidélité des clients est garantie à $90 \%$ près. Certains qualifient même cette clientèle de compartimentée et de sûre. Ils pensent que ceci peut constituer une barrière sérieuse à tout nouveau venu dans le secteur. Une cote moyenne de -4 «significatif» comme faiblesses, est allouée à ce point.

La technologie est le quatrième sujet abordé. Les réponses font ressortir que les coûts de certaines machines sont relativement élevés même si elles sont faciles à trouver. Cependant,

on peut recourir à la sous-traitance pour éviter des investissements coûteux. $90 \%$ des répondants affirment que le recrutement de la main-d'oeuvre est difficile parce que l'ouvrier doit être polyvalent et que dans les régions on n'offre pas de cours dans ce domaine. De plus, à cause de la concurrence très forte pour garder les clients, les prix doivent être très concurrentiels et en conséquence les salaires offerts sont loin d'être attrayants.

Quant à la question sur l'innovation, tous admettent que la réduction des coûts est l'objectif visé afin de pouvoir maintenir la part de marché. Depuis les cinq ou six dernières années, $20 \%$ des entreprises du secteur ont informatisé leur système de comptabilité et de gestion, mais il s'agit des plus anciennes entreprises, donc les plus connues. En général, tous sont d'avis que les salaires constituent 20-25\% des coûts d'opération et que le secteur est capable de rendements d'échelle intéressants avec des équipements modernes mais très chers. De toute façon, toute augmentation de volume ne peut pas viser le marché local déjà saturé. Il faudrait penser à élargir le rayon d'action, mais interviennent alors les coûts de transport qui peuvent se révéler prohibitifs. A ce point on accorde une moyenne de +3 «faiblement significatif», comme forces.

On passe ensuite au cinquième point traitant du gouvernement. En ce qui a trait aux tracasseries généralement reprochées aux gouvernements par les PME, ce secteur n'a mentionné aucune plainte particulière. Toutefois, seulement $30 \%$ des répondants soulignent qu'ils se sont prévalus des subventions offertes par les gouvernements pour la création d'emploi et l'amélioration des immobilisations. Il semble y avoir un manque d'informations concernant les programmes de subven- 
tions gouvernementales. $\grave{A}$ ce point on accorde une moyenne de +2 «mérite considération», comme forces.

Le sixième point du questionnaire traite des produits substituts. On relève qu'il n'y a pas de marque dominante sur le marché des portes et fenêtres. En général, les répondants disent que le marché est bon puisque les clients leur sont très fidèles. Ils mentionnent aussi qu'ils vendent un produit très peu différencié et qu'ils font face à plusieurs concurrents dans la région. Il s'ensuit qu'ils sont tous conscients que l'acheteur a accès à une gamme étendue de produits substituts. Dans aucun cas, on n'a relevé une dissimilitude entre les prix.

Compte tenu de la saturation du marché en terme d'offre, de la faible marge entre le prix de vente et le coût unitaire et ce, à cause de la concurrence, un score moyen de -5 «très significatif», comme faiblesses, est estimé pour ce point.

Le septième point concerne les substituts aux intrants.

Tous les intrants utilisés dans ce secteur, sauf la main d'oeuvre, peuvent se trouver sur le marché en tout temps et en quantité nécessaire. Les fournisseurs de ces intrants (le bois, les vitres, le vinyle, le poly-vinyle, la peinture, la quincaillerie, etc.) sont nombreux. A part la main d'oeuvre, c'est le bois qui est le plus important du point de vue des dépenses relatives.

Toutes choses égales par ailleurs, compte tenu de la facilité d'approvisionnement en intrants autres que la main d'oeuvre, on accorde une note moyenne de +5 «très significatif» à ce sujet.

Le huitième point considère la distribution du produit du centre de fabrication aux points de vente. Tous les répondants affirment qu'ils ont facilement accès aux canaux de distribution que sont les transporteurs et les points de vente. La plupart mentionnent que plus de $50 \%$ de leurs ventes se font directement sur commande par les entrepreneurs ou par les grandes chaînes de magasins ayant une section de quincaillerie. Soulignons que peu de répondants (20\%) pensent au marché de l'exportation comme moyen d'écouler leurs produits. Les autres sont d'avis que le marché local est leur sphère d'activité principale. Une cote moyenne de +2 «mérite considération», est accordée à ce point.

Le dernier point du questionnaire couvre les autres facteurs non encore abordés mais qui peuvent avoir une influence sur la décision finale de l'entrepreneur potentiel.

Les répondants sont unanimes pour dire que les institutions financières sont sensibles à leurs démarches dans la mesure où ils font preuve de solvabilité depuis au moins trois à cinq ans et qu'ils performent sur le marché. Ils mentionnent que pour ceux qui arrivent à se tailler une place dans ce secteur, la compétition se base surtout sur le prix et le maintien d'un inventaire permettant de livrer les commandes dans les délais indiqués. A ceci, ils ajoutent que le contrôle de la qualité est primordiale si l'on veut garder la fidélité de ses clients. 
Ils soulignent aussi que, très dépendant des activités de la construction, ce secteur en connaît les mêmes cycles. En conséquence, plus de $60 \%$ des répondants envisagent l'avenir avec pessimisme à cause des taux d'intérêt élevés qui contribuent à ralentir les activités de mises en chantier et de rénovation domiciliaire.

Quant aux conseils que les répondants donneraient aux éventuels entrepreneurs désireux de s'engager dans se secteur, plus de $60 \%$ croient que même avec la meilleure technologie, une bonne localisation, une qualité de produit supérieure, de la détermination et de la volonté, les chances de réussite sont minces pour ne pas dire nulles. Les raisons avancées sont le caractère cyclique de ce secteur, l'offre qui satisfait très bien la demande, la concurrence très forte et évidemment le ralentissement marqué, depuis quelque temps, des activités de la construction. Ce point a reçu une moyenne de -6 «important», comme faiblesses.

En résumé, si on réunit les différentes cotes accordées aux neuf points touchés par la démarche, on a le résultat consigné dans le tableau 1 ci-après :

Tab. 1 Indice de vulnérabilité

\begin{tabular}{|l|c|c|}
\hline \multicolumn{2}{|c|}{ Forces (moyenne) } & Faiblesses (moyenne) \\
\hline 1) Barrières à l'entrée & +4 & -5 \\
2) Fournisseurs & -4 \\
3) Acheteurs & +3 & \\
4) Technologie & +2 & -5 \\
5) Gouvernements & +5 & \\
6) Produits substituts & +2 & -6 \\
7) Intrants substituts & & -20 \\
8) Distribution & 16 & -4 \\
9) Autres facteurs & & \\
TOTAL moyen & & \\
ECART moyen & & \\
\hline
\end{tabular}

En conclusion, il est risqué de s'aventurer dans ce secteur, du moins pour le moment. En effet, l'écart de -4 indique clairement que les faiblesses dépassent significativement les forces et rendraient le potentiel entrepreneur particulièrement vulnérable aux forces de ce marché.

Le deuxième secteur investigué est celui de l'imprimerie. Les entreprises contactées se rangent plutôt dans la catégorie des petites entreprises où $63 \%$ déclarent avoir un chiffre d'affaires de $250000 \$$ et moins, le reste oscillant autour de 1 à 2 millions de dollars. En ce qui concerne le nombre d'employés, $50 \%$ ont entre 2 et 10 employés, $30 \%$ entre 11 et 20 et $20 \%$ de 20 à 40 employés. 
L'analyse des différentes parties du questionnaire révèle les caractéristiques dominantes de ce secteur. D'abord, en considérant les barrières à l'entrée, les répondants sont tous d'accord pour dire que s'établir dans ce secteur n'est pas difficile, car on peut débuter avec aussi peu que 25000 \$. Mais, à la question de savoir s'il y a possibilité de représailles de la part de ceux déjà établis sur le marché contre les nouveaux venus, les réponses de $70 \%$ de l'échantillon sont positives et mentionnent les coupures dans les prix comme moyen le plus couramment utilisé. Toutefois, toutes les réponses concordent pour dire que la connaissance des facettes du métier d'imprimeur et de bonnes notions de gestionnaire sont un très bon atout. Enfin, $70 \%$ des répondants estiment que le montant d'inventaire à maintenir pour répondre aux commandes urgentes, ainsi que le fonds de roulement requis pour mener à bien les opérations courantes sont minimes, soit de $10000 \$$ en moyenne.

A la lumière des renseignements recueillis, une moyenne de +2 «mérite considération», comme forces, est obtenue à cette première partie du questionnaire.

Le deuxième point traite des fournisseurs. La totalité des réponses souligne qu'ils sont bien approvisionnés de tous les intrants nécessaires. Il y a sur le marché un choix assez varié de fournisseurs offrant des produits substituts. Il n'y a pas de firme dominante parmi les fournisseurs, de sorte que les prix sont très concurrentiels. De même, il n'y a pas de quantité minimale à acheter d'un foumisseur pour obtenir un bon prix. Les répondants relèvent aussi qu'il est très avantageux de se spécialiser dans un champ bien spécifique de l'imprimerie afin de rentabiliser les machines. Un score moyen de +1 , «significatif», comme forces, est accordé à ce point.

En troisième lieu, on considère les acheteurs. Près de $50 \%$ des répondants avancent que la publicité n'est pas nécessaire, mais le reste estime qu'il en faut très peu, car avec du bon travail, le bouche à oreille suffit amplement. Tous disent que 90 $\%$ et plus de leurs clients sont très fidèles. Les produits sont peu différenciés et il suffit de respecter les normes de qualité, d'offrir un service rapide et d'accorder des rabais sur la quantité pour s'attirer des clients et les garder.

Une note moyenne de +2 , «mérite considération» comme forces, est attribuée à cette partie.

Le quatrième point couvre la technologie. L'ensemble des réponses atteste que la main-d'oeuvre est difficileà trouver et qu'elle constitue de 20 à $25 \%$ des coûts. La machinerie est facile à trouver mais coûteuse, si on veut se maintenir à la fine pointe de la technologie. L'innovation basée sur la réduction des coûts est celle poursuivie par les répondants oeuvrant dans ce secteur.

Tous les répondants estiment qu'ils produisent une marchandise de bonne qualité, que l'imprimerie est une activité qui connaît de courts cycles dont les opérations tendent en grande partieà être informatisées, en particulier l'administration, la mise en page et la photocomposition. Ils mentionnent aussi que les rendements d'échelle caractérisent la production des travaux d'imprimerie. 
A cause de la rareté de la main-d'oeuvre spécialisée, du coût élevé de la modernisation, un score moyen de -4 «significatif» comme faiblesses, est porté au compte de ce sujet.

Les gouvernements forment le cinquième problème considéré dans le questionnaire. Les tracasseries gouvernementales ne sont pas considérées comme un handicap au développement d'une entreprise d'imprimerie. Pour encourager «l'employabilité», le gouvernement accorde des subventions qui, selon près de $40 \%$ des répondants, ne sont pas aisées à obtenir. Une note moyenne de +2 «mérite considération» comme forces est accordée à cette question.

Le sixième sujet traité considère les substituts aux produits. Même si les répondants considèrent qu'il y a plusieurs concurrents oeuvrant sur le marché, la demande pour les produits d'imprimerie est jugée bonne. L'information sur les stratégies des concurrents en termes de variantes du produit est facile à obtenir et ces variantes sont relativement simples à imiter. Tout le monde est optimiste et personne n'entrevoit de ralentissement futur du secteur. Un score moyen de +2 «mérite considération» est attribué à ce sujet.

Les substituts aux intrants forment la septième partie du questionnaire. L'intrant variable le plus important est, après la main d'oeuvre, le papier qui représente $80 \%$ des coûts variables. A cause de la diversité des fournisseurs, les produits substituts sont nombreux et disponibles en tout temps. Aucun des répondants n'a souligné de difficultés particulières reliées aux intrants ou à leurs substituts. En conséquence, une moyenne de +3 «faiblement significatif» est donnée à ce point.

Le huitième sujet abordé est la distribution. En ce qui concerne ce point, l'imprimerie semble très peu affectée, puisque c'est une industrie qui travaille sur commande à $90 \%$ de son chiffre d'affaires. Les clients vont à l'imprimeur. Bien sûr, celui-ci délègue, à l'occasion, des représentants auprès des clients potentiels pour les informer des nouvelles possibilités de l'entreprise et il s'occupe aussi de la livraison. Les répondants mentionnent également qu'ils comptent aussi beaucoup sur les pages commerciales de l'annuaire téléphonique pour se faire connaître. A cet aspect est accordé un score moyen de +1 comme forces.

Le dernier thème envisagé couvre les autres points d'intérêt pour le secteur. Près de $90 \%$ disent que les relations avec les institutions financières sont bonnes dans la mesure où on a des garanties à offrir ou qu'on a fait ses preuves. C'est un secteur où le syndicalisme est absent, sauf dans les très grandes entreprises. A peu près $50 \%$ des répondants mentionnent l'aspect saisonnier de l'entreprise; les autres, au contraire, soulignent que la production est stable et assez régulière. La production se fait sur commande et selon des spécifications précises. D’ailleurs, tous les répondants sont unanimes à relever que la force d'une entreprise repose largement sur sa capacité à exécuter promptement et fidèlement les instructions du client. La majorité des répondants (80\%) juge le marché plutôt bon. Les journaux, les revues spécialisées et les représentants des fournisseurs sont les principales sources d'information sur les 
développements technologiques touchant l'industrie. A peu près le même pourcentage affirme qu'ils ne font pas de planification à long terme. On ne peut planifier plus de deux ans à l'avance, probablement à cause de la sensibilité du secteur aux changements technologiques. En ce qui concerne la localisation de l'entreprise, par rapport au marché du produit ou à celui des intrants, seulement $30 \%$ des répondants disent lui accorder de l'importance. Plus de $60 \%$ d'entre eux évaluent le taux de croissance des entreprises du secteur à 4 ou $5 \%$ annuellement. La concurrence se situe surtout au niveau des prix, d'où la nécessité de réduire le plus possible les coûts. Certains (25\%) estiment que le nombre d'entreprises commence à dépasser le seuil nécessaire et suffisant pour satisfaire la demande. Mais la majorité (80\%) croient que la qualité du produit, l'introduction d'équipements modernes performants, le respect des délais de livraison sont les meilleures armes contre la compétition alimentée par le travail au noir des très petites unités. Ce point est coté par une moyenne de +2 «mérite considération», comme forces.

Les scores moyens consignés aux différents facteurs considérés sont réunis dans le tableau 2 qui suit.

Tab. 2 Indice de vulnérabilité

\begin{tabular}{|l|c|c|}
\hline \multicolumn{2}{|c|}{ Forces } & Faiblesses \\
\hline 1) Barrières à l'entrée & -2 & \\
2) Fournisseurs & +1 & \\
3) Acheteurs & +2 & -4 \\
4) Technologie & +2 & \\
5) Gouvernements & +2 & \\
6) Substitution (produits) & +3 & \\
7) Substitution (intrants & +1 & \\
8) Distribution & +2 & \\
9) Autres & +15 & -4 \\
TOTAL moyen & +11 & \\
ÉCART moyen & & \\
\hline
\end{tabular}

En supposant que le potentiel entrepreneur s'y connaît dans le métier de l'imprimerie, qu'il dispose d'une moyenne de 20000 \$ liquide et est armé de bonnes notions de gestion ou à défaut qu'il peut s'adjoindre un associé gestionnaire, il peut décider, en considérant le résultat de l'écart, de se lancer dans le secteur de l'imprimerie. Il a toutes les chances d'évoluer et de connaitre une bonne croissance maintenant qu'il est bien conscient de ses forces, de ses faiblesses et des particularités essentielles du marché, qu'elles soient en amont, en aval ou au niveau de la production. 


\section{Conclusion}

Cette étude tente de démontrer à certains types d'entrepreneurs potentiels définis dans le texte, comment, à l'aide d'une démarche dite de pré-démarrage, ils peuvent mieux évaluer les caractéristiques de leur environnement et, ce faisant, augmenter leur chance de survie après le démarrage. Une telle démarche ne doit pas être confondue avec les traditionnelles études de marché ou celles dites de «faisabilité». Elle s'en différencie, car elle force l'entrepreneur potentiel à se réaliser en appréciant son potentiel, sans négliger son imagination, face aux forces et faiblesses des différentes composantes de son environnement. En identifiant les caractéristiques de son milieu d'évolution, il peut se préparer plus adéquatement, soit par des stratégies plus rodées, soit par l'acquisition de nouvelles ressources.

Cette approche n'écarte en rien les idées d'innovation ou d'invention que l'entrepreneur croit posséder et pense pouvoir mettre à profit dans son entreprise. A chaque étape de l'analyse, il déterminera comment et à quel niveau il peut utiliser son imagination, source d'idées d'invention et d'innovation tant pour contourner une difficulté prévue, pour découvrir un nouveau créneau que pour mieux satisfaire les besoins de la clientèle visée. De toute façon, les idées porteuses d'invention ou d'imagination interviendront sûrement dans l'analyse, et surtout lors de la pondération, en faveur ou contre les questions envisagées.

Dans toute prise de décision, où des pondérations doivent être attribuées, il faut bien sûr tenir compte des jugements de valeur qui s'apparententà la détermination d'une probabilité subjective. Le décideur doit se rappeler cependant que c'est lui, en définitive, qui doit payer le coût d'une décision basée sur une analyse émotive et une pondération biaisée. Aussi, tout en accordant une place convenable à l'intuition et à l'imagination, il doit s'asurer que, dans les limites de l'information disponible, les conséquences de toutes les étapes intermédiaires franchies soient clairementétablies, de sorte qu'il soit en mesure d'évaluer les coûts, les bénéfices et les effets sur la décision finale qu'il aura à prendre.

En considérant l'imprimerie et les portes et fenêtres, deux secteurs différents selon certaines caractéristiques, cette approche a permis de déterminer l'étendue des risques à encourir en prenant la décision de démarrer ou non une entreprise. Le modèle de diagnostic de "pré-démarrage» développé dans cette recherche a permis à l'entrepreneur potentiel d'établir que, compte tenu de ses ressources personnelles, s'aventurer dans l'imprimerie est moins risqué que dans la fabrication des portes et fenêtres. En fait, pour ouvrir une imprimerie, le capital fixe et de fonctionnement nécessaires sont peu élevés relativement à ceux requis pour les portes et fenêtres. Le secteur des portes et fenêtres est, quant à lui, très vulnérable aux cycles économiques et manifeste des tendances à la concentration forçant les plus fragiles à disparaître. Dans l'imprimerie, le démarrage peut s'amorcer dans un sous-sol avec un capital modeste. 
Selon les chiffres disponibles de Statistique Canada pour les industries, de 1984 à 1986 le nombre d'entreprises fabriquant des portes et fenêtres au Québec est passé de 172 à 174, soit une augmentation négligeable de 1\%. C'est un secteur stable où les entrées sont rares et difficiles à cause des raisons évoquées précédemment. Pour la même période, le nombre d'établissements d'imprimerie est passé de 796 à 855 , soit une croissance de près de $8 \%$. Selon les répondants, le taux de disparition de ce type d'entreprises dans la région est inférieur à celui des entrées. Ce qui est conforme aux résultats de notre enquête.

Généralement, on peut supposer que la perception qu'a une entreprise existante sur l'entrée éventuelle d'un nouveau concurrent sera défavorable et que, dans ce cas, les réponses obtenues sont biaisées. Tel ne semble pas être le cas, cependant, car des répondants indépendants, comme les vendeurs et le gérant d'une banque faisant affaire avec ces deux secteurs, confirment les dires des entrepreneurs consultés. En fait, en 1989-1990, une nouvelle imprimerie a pris naissance dans la région et aucune entreprise de portes et fenêtre depuis 1985 n'y est signalée.

L'apport de cette approche, si elle est bien menée et si son adoption et son application sont encouragées par tous les intervenants concernés par la création et la survie des PME, contribuera d'une façon non négligeable à décroître le taux de mortalité des PME et PE et, de ce fait, à réduire les coûts sociaux qu'impliquent pour la société les politiques actuelles d'encouragement mises de l'avant à leur intention.

\section{Bibliographie}

Aldrich, H. et Herker, D., (1977), «Boundary shanning roles and organization structure», Academy of Management Review, avril, p. 218-230.

Arena, R. et al., (1988), «Filières et découpages productifs», Traité d'économie industrielle, Economica, p. 249.

Boag, A.D. et Dastmalchian, A., (1988), «Market vulnerability and the design and management of the marketing functions in small firms", Journal of Small Business Management, octobre, p. 37-43.

Chaganti, R., Chaganti, R. et Vijay, M., (1989),.«Profitable small business strategies under different types of competition», Entrepreneurship Theory and Practice, printemps, p. 2135.

Cool, K., Dierickx, I.. et Jenison, D., (1989), «Business strategy, market structure and riskreturn relationships : a structural approach», Strategic Management Journal, vol. 10, p. 507-522.

Covin, G.J. et Slevin, P.D., (1989), «Strategic management of small firms in hostile and benign environments», Strategic Management Journal, vol. 10, p. 75-87.

Dollinger, M.J., (1985), «Environmental contacts and financial performance of the small firm», Journal of Small Business Management, janvier, p. 25-30.

El-Ansary, A. I. et Stern, L.W., (1972), «Power measurement in the distribution channel», Journal of Marketing Research, vol. IX, février, p. 47-52. 
Finley, L., (1984), «Can your small company acquire resources as favorably as the large company?», American Journal of Small Business, vol. IV, $\mathrm{n}^{\circ} 1$, été, p. 19-25.

Fourcade, C., (1986), «Le démarrage des entreprises : une comparaison internationale», La PME dans un monde en mutation, sous la direction de P.A. Julien, J. Chicha et A. Joyal, p. 253-277.

Galbraith, C.S. et Stiles, C.H., (1983), «Firms profitability and relative firm power», Strategic Management Journal, vol. 4, p. 237-249.

Garrouste, P.,(1988), «Filière et technologie»,Traitéd' économie industrielle, Paris, Economica, p. 237.

Jacquemin, A. et Rainelli, M., (1984), «Filières de la nation et filières de l'entreprise», Revue Economique, $\mathrm{n}^{\circ}$ 2, mars, p. 379-389.

Jain, S.C., (1979), «Self-appraisal and environmental analysis in corporate planning», Managerial Planning, janvier-février, vol. 27, $n^{\circ} 4$, p. 16-28.

Jorgensen, J. et Fautska, T., (1978), QUID-How to make the Best Decisions of your Life, New York, Walker and Co. Publishers.

Lambkin,M.,(1988), «Order of entry and performance in new markets», Strategic Management Journal, vol. 9, p. 127-140.

Le Québec mis en chiffres, Statistiques sociales et économiques sur les régions et les villes du Québec, L'économie 1988.

Malsot, J., (1980), «Filières et effets de domination dans le système productif», Annales des Mines, janvier, p. 29-40.

Moody, Paul E., (1983), Decision Making, Proven Methods for Better Decisions, New York, McGraw Hill Company.

Pearce II, J.A. et Robinson Jr, R.B., (1985), Strategic Management, Strategy, Formulation et Implementation, New York, McGraw Hill, $2{ }^{\mathbf{e}}$ édition.

Porter, M.E., (1980), Competitive Strategy, New York, The Free Press.

Porter, M.E., (1985), Competitive Advantage, New York, The Free Press

Scherer, F.M.,(1980), Industrial Market Structure and Economic Performance, Chicago, Rand McNally.

Statistique Canada, (1987), nos 31-203, 36-251 et 35-250.

Stevenson, H.H. et Jarello, J.C., (1990), «A paradigm of entrepreneurship : entrepreneurial management», Strategic Management Journal vol. 11, p. 17-27.

Tollet, R., (1982), «Approches méthodologiques de la politique industrielle au travers du concept de filière», Cahiers Economiques de Bruxelles, $\mathrm{n}^{\circ} 96,4^{\mathrm{e}}$ trimestre.

Torre, A., (1988), «Une approche industrielle des déstructurations internes des processus de production», Revue d'Economie urbaine et régionale, $\mathrm{n}^{\circ} 3$, p. 413-430.

Yon, B., (1983), «Structure des filières et stratégie des firmes», Économies et Sociétés, tome XVII, $n^{\circ}$ 5, mai, p. 822-837.

Zimmermer, T.W. et Scarborough, N.H., (1988), Small Business Fundamentals, Columbus, Ohio, Merrill, 496 p. 